University of Rhode Island

DigitalCommons@URI

Mechanical, Industrial \& Systems Engineering

Faculty Publications

$9-13-2020$

\title{
Fatigue Damage of a Single-Edge Notched Beam Specimen Under Variable Amplitude Loading with Similar Probabilistic and Cycle Counting Statistics
}

Hewenxuan Li

David Chelidze

University of Rhode Island, chelidze@uri.edu

Follow this and additional works at: https://digitalcommons.uri.edu/mcise_facpubs

The University of Rhode Island Faculty have made this article openly available.

Please let us know how Open Access to this research benefits you.

This is a pre-publication author manuscript of the final, published article.

Terms of Use

This article is made available under the terms and conditions applicable towards Open Access

Policy Articles, as set forth in our Terms of Use.

\section{Citation/Publisher Attribution}

Li H., Chelidze D. (2021) Fatigue Damage of a Single-Edge Notched Beam Specimen Under Variable Amplitude Loading with Similar Probabilistic and Cycle Counting Statistics. In: Kerschen G., Brake M.R., Renson L. (eds) Nonlinear Structures \& Systems, Volume 1. Conference Proceedings of the Society for Experimental Mechanics Series. Springer, Cham. https://doi.org/10.1007/978-3-030-47626-7_44

This Article is brought to you for free and open access by the Mechanical, Industrial \& Systems Engineering at DigitalCommons@URI. It has been accepted for inclusion in Mechanical, Industrial \& Systems Engineering Faculty Publications by an authorized administrator of DigitalCommons@URI. For more information, please contact digitalcommons-group@uri.edu. 


\title{
Fatigue Damage of a Single-Edge Notched Beam Specimen under Variable Amplitude Loading with Similar Probabilistic and Cycle Counting Statistics
}

\author{
Hewenxuan Li and David Chelidze \\ Nonlinear Dynamics Laboratory, \\ Department of Mechanical, Industrial and Systems Engineering, \\ University of Rhode Island, Kingston, RI 02881, USA
}

\begin{abstract}
Fatigue life estimation under variable amplitude (VA) loading is still a major unresolved engineering problem. The linear cumulative damage rule (LDR) based methodology is inadequate to predict fatigue damage or useful remaining life if the fatigue loading is complex (not sinusoidal). In addition, the LDR based damage estimation methods rely on statistics of various cycle counting methodologies (e.g., rain-flow counting) where the load interaction effects are being ignored. It has been shown that the damage estimation fails if two load-time (load-cycle) histories with different temporal dynamics have the same or similar load spectra. A robust damage estimation should take the temporal dynamics, i.e., the overload/underload information, stress memory statistics, into consideration. In this extended abstract, the shortcomings of the LDR based methods will be presented through a comparison where two VA load spectra with similar stress history and cycle counting statistics were applied to a singled-edge notched beam specimen.
\end{abstract}

Keywords: Fatigue crack propagation, Fatigue damage estimation, Rain-flow counting, Variable amplitude loading, Load statistics

\section{INTRODUCTION}

Fatigue damage estimation under constant amplitude (CA) loading is a solved engineering problem while damage estimation under highly irregular loading is still an open field of study among researchers in fatigue structure and materials. Among existing methodologies in predicting useful remaining life of engineering structures, linear cumulative damage rules (LDR) and linear fracture mechanics based empirical crack propagation laws are widely used in contemporary industry for their simplicity and general accuracy. The LDR, or Palmgren-Miner's rule tries to resolve the damage under VA loading by consider damage as a summation of the fractions between the number of applied cycles in a stress range and the number of cycles to failure under CA loading with the same stress range [1], or simply

$$
D=\sum_{i=1}^{n} \frac{n_{i}}{N_{i}}
$$

where $D$ is the estimated damage, $n_{i}$ is the number of cycles applied at the $i$ th stress range, $N_{i}$ is the number of cycles to failure at the same stress range under CA loading (this number is obtained from the S-N curve of the material under investigation). However, these methodologies fails to estimate the damage or of the useful remaining life when the applied loads are complex or have variable amplitude. This is partly due to the load interaction effect introduced by crack closure and opening at the wake of the crack [2]. Later on, modifications have made to these models by 1) adding weights to each binned load level in LDR and 2) adding additional empirical parameters into the Paris law based method in order to take load interaction effect into consideration [3]. Here, we focus on the modification to the LDR based methodologies for its simplicity in computation. The accuracy of damage estimation decreases dramatically when various applied loads have similar statistical characteristics but different temporal dynamics due 

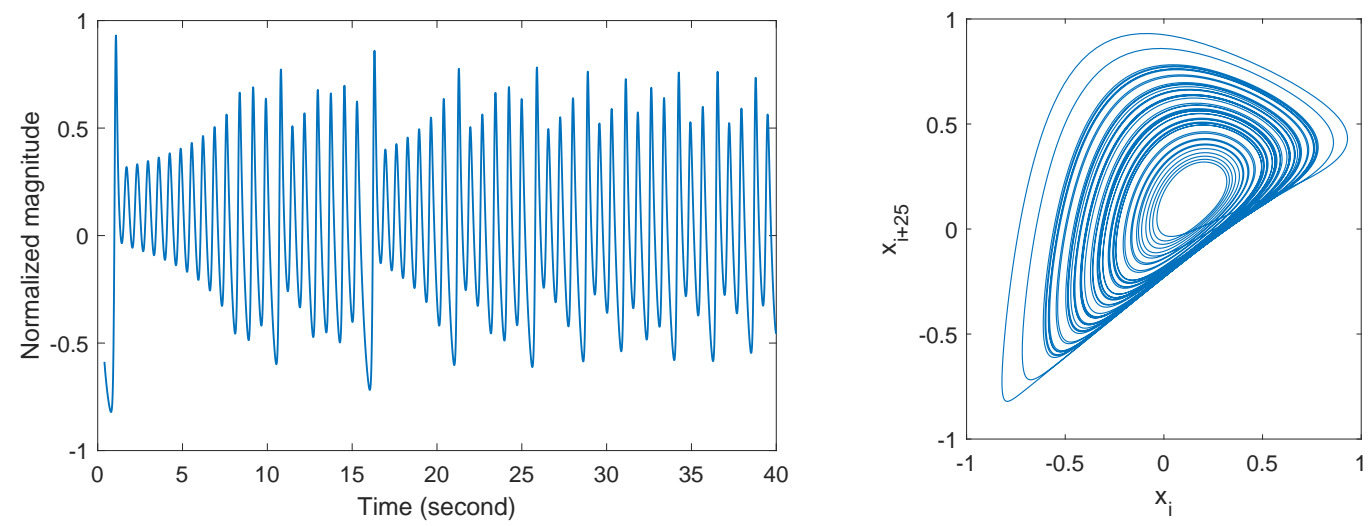

Fig. 1. Upper two: pressure-voltage curve by incremental increasing the voltage input to the regulator; upper left: pressure-voltage curve for the front regulator; upper right: rear regulator. Lower two: deflection-pressure curves and the corresponding curve fitting results.

to its simple dependence on stress range statistics $[1,4]$. For example, these applied loading could have similar power spectral density, probability density (or mass), and/or cycle counting statistics. According to previous experimental work [5], the fatigue lives on the same notched beam structure have two times the difference between chaotic loading and its corresponding random surrogate with nearly identical magnitude statistics and power spectral density. Additionally, simulation based study indicates that the damage estimation fails if two load-time (load-cycle) histories with different temporal dynamics have the same or similar load spectra [6]. This indicates that metric based on simple spectral or statistical parameters tend to fail the purpose of accurately estimate damage. In the following section, a demonstrative simulation will be presented to show the insufficiency of the current LDR based methodologies.

\section{FATIGUR CRACK PROPAGATION SIMULATION}

\section{SIMULATION SETUP}

In order to illustrate the shortcomings of the LDR and its modifications, an illustration based on fatigue crack propagation simulation was conducted.

In order to keep the stress level statistics and cycle counting statistics of loading consistent, a normalized load time history was obtained by numerically integrating the Lorenz equation [7]:

$$
\left\{\begin{array}{l}
\dot{x}=10(y-x) \\
\dot{y}=-x y+28 x-y \\
\dot{z}=x y-\frac{8}{3} z
\end{array}\right.
$$

where $\left({ }^{*}\right)$ represents derivative with respect to time. And the solution was obtained using a sampling frequency of $250 \mathrm{~Hz}$ and the initial condition of $x_{0}=0, y_{0}=-0.01, z_{0}=27$. For simplicity, the solution to the third variable, $z$, was selected due to its wide sense stationarity. The obtained load time history was normalized in its magnitude and its corresponding phase space representation can be obtained through phase space reconstruction, see Fig. 1.

In order to fit the load time history to the LDR, local maximum stress and minimum stress were extracted to form the fatigue spectrum of the obtained chaotic load time history, and it was name as the chaotic reversals or the chaotic spectrum. Since the comparison was based on similar stress history statistics and cycle counting statistics, the obtained stress range of the chaotic reversals can be defined as:

$$
\Delta \sigma^{i}=\sigma_{\max }^{i}-\sigma_{\min }^{i}
$$

where, $i$ indicates the $i$ th load cycle range, please refer to Fig. 2 for detailed explanation. The previously defined load cycle range (or stress range), $\Delta \sigma^{i}$, which is considered as a major driving force of macro fatigue cracks was 


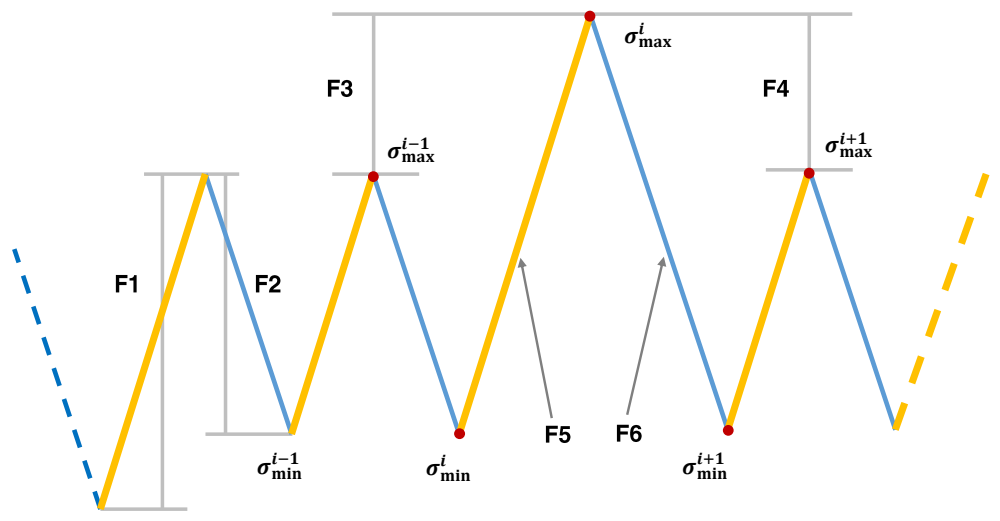

Fig. 2. Schematics of the load factors that are used in this report. F1: load cycle range; F2: unload cycle range; F3: negative peak difference; F4: positive peak difference; F5: load cycle range that corresponds to its following overload, F4; F6: unloading cycle range that corresponds to it following overload, F4.
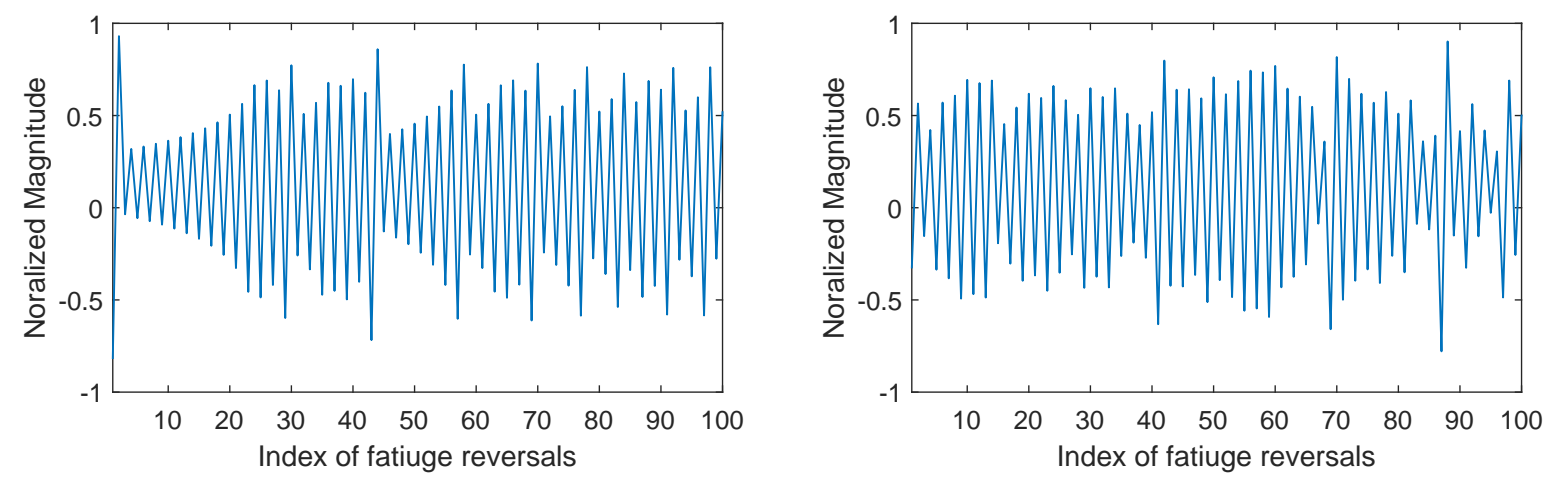

Fig. 3. Load-cycle history between the chaotic spectra and the random spectra. Left: the chaotic spectra; right: the random spectra.

randomly permuted temporally. For wide sense stationary load time histories, the cycle counting statistics and the stress level statistics should be identical to its chaotic counterparts. Here, rain flow counting was selected among various cycle counting methods since it accounts for the load interaction by taking the stress-strain memory effect into consideration $[8,9]$. The obtained chaotic spectrum and its randomly permuted version, called the random spectrum, will be used as input spectra to a widely used fatigue crack propagation software package, AFGROW 5.3. As a direct comparison, from Fig. 3, the two spectra have different temporal dynamics. However, the stress level statistics, measured by the stress level counting, and the rain flow cycle counting statistics, measured by cycle range counting are identical among the two cases, see Fig. 4.

After the preparation of the two fatigue spectra, crack propagation simulations will be performed in AFGROW. The specimen is assumed to be made from 2024-T3 aluminum alloy. According to the nature of the applied load from the fatigue experimental apparatus and the specimen configuration, single-edge through crack was considered under pure bending. Since both the chaotic and random spectra are normalized in their magnitude, AFGROW considers it as $1 \mathrm{ksi}$. Therefore, in order to fulfill the purpose of simulating crack propagation under realistic loading, the load spectra are multiplied with a spectrum multiplication factor[10] (SMF) of 14 (maximum stress within 14 ksi). Further, since the simulation should take load interaction effects into account, a simple crack retardation model [11], crack closure model, was utilized. The initial crack length was set to 0.3 inches and the criteria for stopping the simulation are 1) crack size reaches 0.6 inches and 2) stress intensity factor reaches the fracture toughness. 

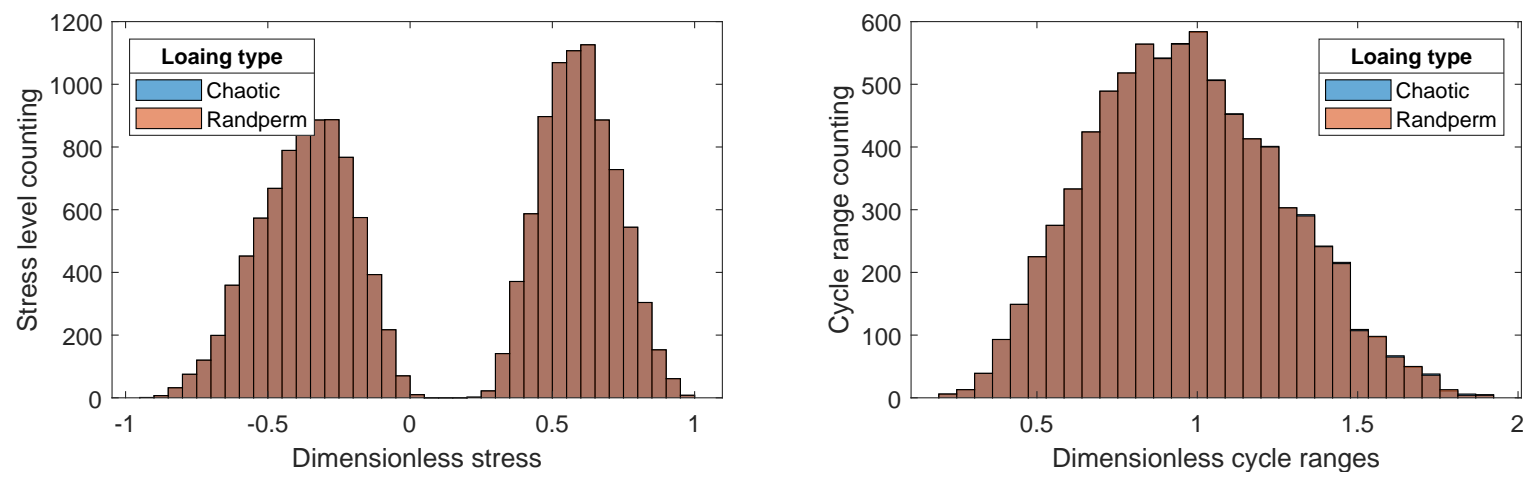

Fig. 4. Comparison of the stress level statistics and the cycle counting statistics between the chaotic spectra and the random spectra. Left: the stress level counting histogram; right: the cycle range counting histogram.

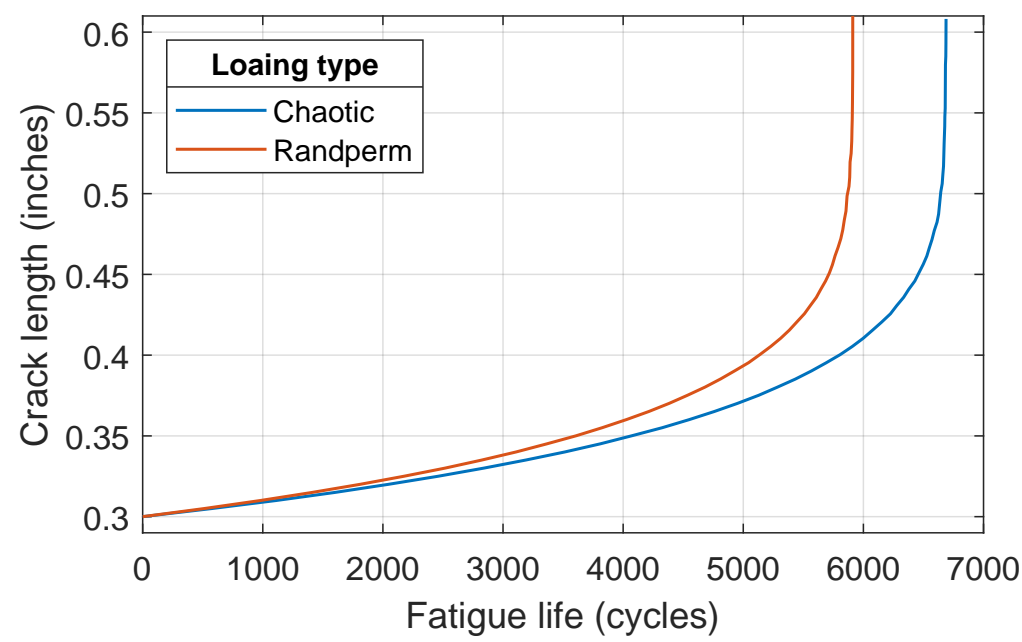

Fig. 5. Comparison between the fatigue life (in applied stress cycles) under chaotic spectra and the random spectra. Left: the crack propagation curve under chaotic spectra loading; right: the crack propagation curve under random spectra loading.

\section{SIMULATION RESULTS}

As it can be observed from Fig. 5, the predicted results indicates the fatigue life, under two 1) similar stress level statistics and 2) similar cycle counting statistics yielded different fatigue life. This difference in fatigue life is assumed to be resulted from the difference in their temporal dynamics since the load sequence has changed considerably after random permutation of the load cycle ranges. This will contradict the damage estimation if LDR is used since the two load spectra (i.e., the chaotic spectrum and the random spectrum) are identical.

\section{DISCUSSION}

This work is aimed to illustrate the importance of taking more sophisticated consideration during fatigue life prediction or damage estimation under VA loading. More specifically, the measures of temporal dynamics By only taking stress level related statistics and/or whenever cycle-counting method is utilized, LDR based methods tend to produce erroneous results.

\section{FUTURE WORK}

For the current work, the load spectrum was selected to be wide sense stationary, this is partly due to the future plan for experimental verification work on the fatigue experiment apparatus in our laboratory environment. Later on, 
simulation-based analysis to a wider range of fatigue load spectra will be conducted to generalize the understanding of the proposed fatigue estimation method. Aside from the simulation, modification to the current experimental setup will be made to assist the findings from simulations.

\section{ACKNOWLEDGEMENT}

This work is supported by the National Science Foundation Grant No. 1561960.

\section{REFERENCES}

[1] J. Schijve, Fatigue of structures and materials. Springer Science \& Business Media, 2001.

[2] S. Suresh, "Fatigue crack deflection and fracture surface contact: micromechanical models," Metallurgical Transactions $A$, vol. 16, no. 2, pp. 249-260, 1985.

[3] E. Santecchia, A. Hamouda, F. Musharavati, E. Zalnezhad, M. Cabibbo, M. El Mehtedi, and S. Spigarelli, "A review on fatigue life prediction methods for metals," Advances in Materials Science and Engineering, vol. 2016, 2016.

[4] S. Suresh, Fatigue of materials. Cambridge university press, 1998.

[5] S. H. Nguyen, M. Falco, M. Liu, and D. Chelidze, "Different fatigue dynamics under statistically and spectrally similar deterministic and stochastic excitations," Journal of Applied Mechanics, vol. 81, no. 4, p. 041004, 2014.

[6] C. D. Li H., "Identification of variable amplitude fatigue loading based on bivariate probability mass functions," in IDETC/CIE 2019 - MSNDC. ASME, 2019.

[7] J. C. Sprott, Chaos and time-series analysis. Citeseer, 2003, vol. 69.

[8] ASTM, "Standard practices for cycle counting in fatigue analysis, e1049 - 85(2017)," 2017.

[9] R. Sunder, S. Seetharam, and T. Bhaskaran, "Cycle counting for fatigue crack growth analysis," International Journal of Fatigue, vol. 6, no. 3, pp. 147-156, 1984.

[10] J. A. Harter, "Afgrow users guide and technical manual," LexTech Inc., Tech. Rep., 2019.

[11] W. Elber, "The significance of fatigue crack closure," in Damage tolerance in aircraft structures. ASTM International, 1971. 